\title{
ESTUDIO ETNOBOTÁNICO DE LA ISLA KALAU, TERRITORIO ANCESTRAL KAWÉSQAR, REGIÓN DE MAGALLANES, CHILE
}

\author{
ETHNOBOTANICAL SURVEY OF KALAU ISLAND, ANCESTRAL \\ KAWESQAR TERRITORY, MAGALLANES REGION, CHILE
}

\author{
Erwin Domínguez ${ }^{1}$, Oscar Aguilera ${ }^{2}$, Rodrigo Villa-Martínez ${ }^{3}$, \\ Juan Carlos Aravena ${ }^{3} \&$ Juan Marcos Henríquez ${ }^{4}$
}

\begin{abstract}
This work documents the vascular flora of Kalau island, located in the Bernardo O'Higgins National Park, protected area within the Kawésqar ancestral territory. This is a first approach to the available ethnobotanical use by the Kawésqar people of vascular plants from Kalau Island. We register 80 vascular plants; 79 of them are native (99\%), with 29 being endemics for Chile and Argentina. Main uses are: 43\% food; $30 \%$ building material for huts, canoes, ropes, and containers; $21 \%$ fuel; and $13 \%$ medicinal. Drymis winteri is the plant species with more registered uses. The most common medicinal use cited is wound healing.
\end{abstract}

Key words: Flora of Chile, Vascular Plants, Medicinal plants, Kawésqar people.

\section{RESUMEN}

Este trabajo documenta la flora vascular de la isla Kalau, ubicada en el Parque Nacional Bernardo O'Higgins, área protegida inserta en el territorio ancestral Kawésqar. Se establece una primera aproximación con respecto a la información etnobotánica disponible sobre el uso y la aplicación de las plantas por los Kawésqar en la isla Kalau. Registramos 80 plantas vasculares, de las cuales 79 son nativas (99\% especies), 29 de ellas endémicas para Chile y Argentina. Los principales usos son: 43\% es comestible, $30 \%$ se aplica a fines de construcción como materia prima en la elaboración de viviendas, canoas, sogas, recipientes y cestas, un $21 \%$ como combustible y $13 \%$ medicinal. Drimys winteri es la especie que presenta la mayor cantidad de usos. Las propiedades medicinales más citadas son cicatrizantes para heridas.

Palabras clave: Flora de Chile, Plantas Vasculares, Plantas medicinales, Etnia kawésqar.

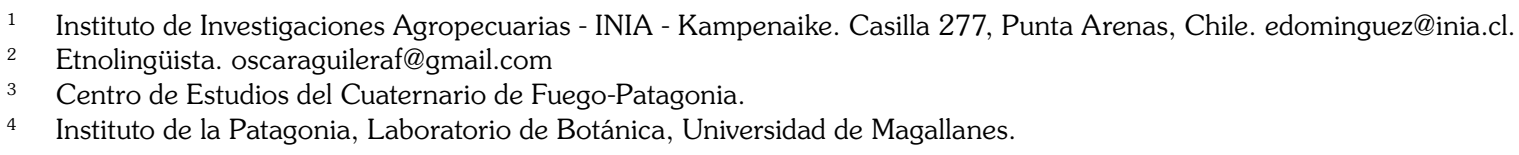




\section{INTRODUCCIÓN}

La isla Kalau ha sido un territorio ancestral para la etnia kawésqar, que ha proporcionado sitios de pesca, caza y recolección de frutos y de plantas medicinales. Representa una de las áreas más expuestas a la zona oceánica, en medio del territorio denominado Málte por el pueblo kawésqar, en el sector occidental del Parque Nacional Bernardo O'Higgins (Aguilera \& Tonko, 2011). El análisis de la flora presente en esta región geográfica nos permite conocer la rareza y singularidad florística de este territorio, así como establecer comparaciones ecológicas con otras áreas y conocer el uso dado por los pueblos originarios.

La presencia de los kawésqar en el extremo sur del país está atestiguada tanto por el territorio que ocupaban como por la toponimia. En el caso del territorio que se extiende desde el Golfo de Penas hasta el estrecho de Magallanes, se han encontrado sitios arqueológicos en las partes noroccidental y meridional de la Patagonia occidental, cuya antigüedad data de entre los 2.000 y 5.000 años (Aguilera \& Tonko, 2011).

La isla Kalau e islas adyacentes (Fig. 1) era escala obligatoria para los kawésqar en los despla- zamientos a través del territorio en su vida nómada, por encontrarse en un entorno donde la caza y recolección estacional era abundante. Desde allí como campamento base, podían acceder a las loberías en tiempo de crías y a los lugares donde era posible recolectar huevos y cazar aves en las temporadas respectivas (Aguilera \& Tonko, 2011).

El objetivo de este trabajo es determinar la flora vascular, y su relación con el pueblo Kawésqar, mediante la confección de un catálogo de sus especies con información de su distribución, estado de conservación, ambiente y usos.

\section{MATERIALES Y MÉTODOS}

\section{Área de Estudio}

Kalau (49²4'25.45” S, 75²9'51.68” O, Fig. 1) es una isla expuesta al Océano Pacíico que se ubica en la porción central del extenso territorio que va desde el Golfo de Penas al estrecho de Magallanes. Esta ubicación en el centro de esta porción del archipiélago patagónico de más de $800 \mathrm{~km}$ en el sentido norte-sur, que el pueblo kawésqar habita desde hace al menos 5 mil años, le da a la isla Kalau una posición estratégica en los recorridos de este

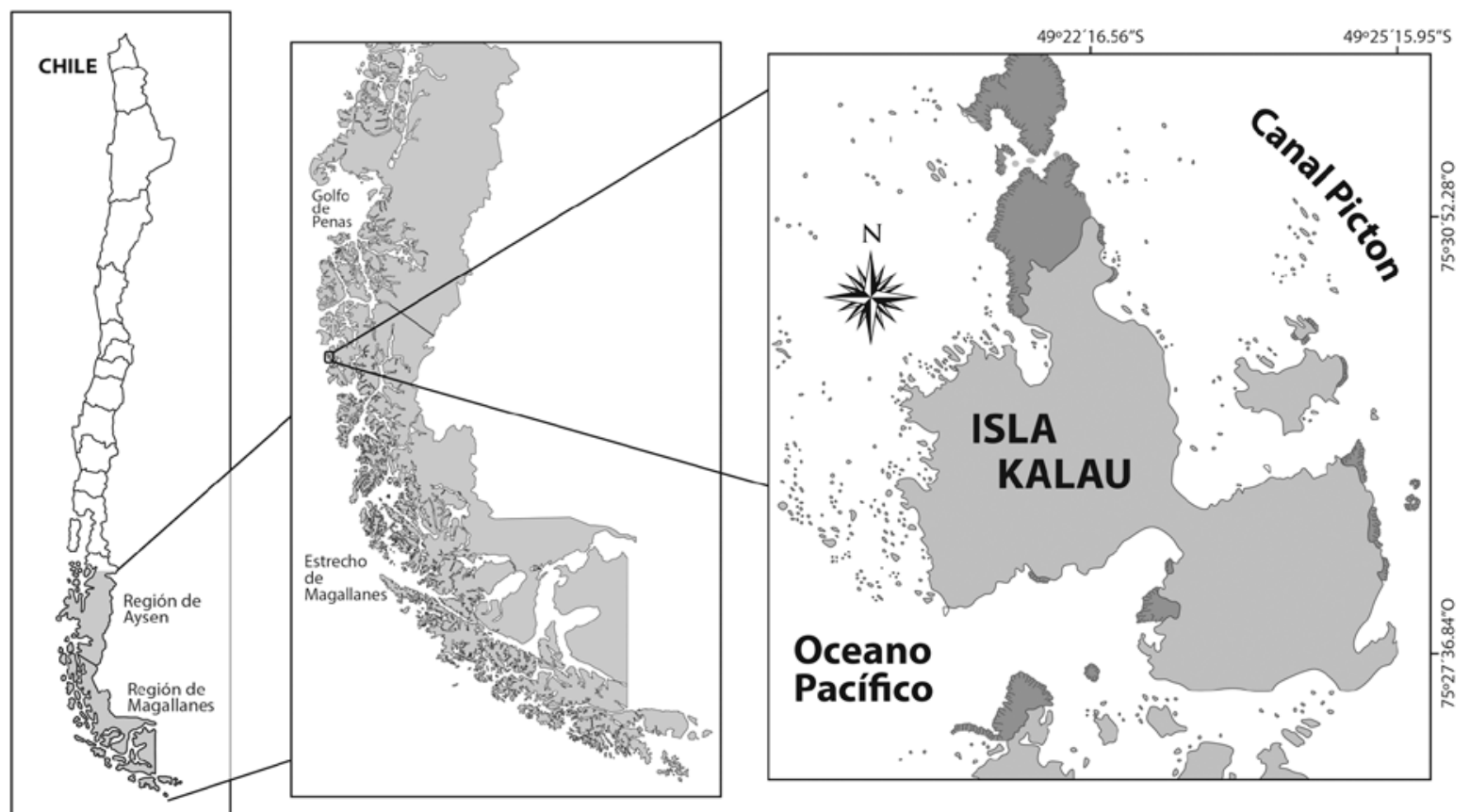

Fig. 1. Localización geográfica de la isla Kalau. 
pueblo canoero por los canales de la región. La isla tiene una superficie de 1.621 ha, y se encuentra a una distancia de $130 \mathrm{~km}$ del Campo de Hielo Sur y a $86 \mathrm{~km}$ del canal Jerónimo, principal ruta marítima (Fig. 1).

En términos de su geología, la isla Kalau forma parte del Complejo Acrecionario Madre de Dios (CAMD), y dentro de éste a la subunidad litoestratigráfica Complejo Duque de York (CDY), con rocas de edad Pérmico Temprano (Forsythe \& Mpodozis, 1979). Esto significa que las rocas que componen la isla son el resultado de un proceso geológico en el que un terreno exótico proveniente del noroeste se habría adherido al margen occidental de Gondwana, representado en este caso por la placa sudamericana, por procesos de subducción, hace aproximadamente 190 millones de años (Forsythe \& Mpodozis 1979, 1983; Hervé et al. 2003; Sepúlveda et al. 2007; Urresty, 2010¹).

La geomorfología de la Isla Kalau corresponde a terrenos de topografía plana con una altura promedio sobre el nivel medio del mar de aproximadamente $5 \mathrm{~m}$ y con alturas máximas no superiores a los 10 $\mathrm{m}$ snm, representadas por promontorios rocosos aislados. Todo este sistema corresponde a la ladera occidental de los sistemas montañosos que predominan hacia el interior del archipiélago patagónico. $\mathrm{Su}$ principal característica es estar formada por lomas bajas que bordean las costas más expuestas al Océano Pacífico. (Urresty, 2010)

La isla Kalau se encuentra en la región con el mayor predominio de los vientos del oeste (westerlies) lo que determina niveles de precipitación que están entre los más altos del planeta. Los vientos oeste suelen ser más fuertes durante el verano y con predominio del norte-noroeste (Carrasco et al. 2002). La precipitación anual resultante en la zona occidental del archipiélago patagónico, donde se ubica la isla Kalau, excede los $7 \mathrm{mil} \mathrm{mm}$ (DGA, 1987), la que se distribuye homogéneamente durante el año. La temperatura del aire, con un promedio anual de alrededor de $8{ }^{\circ} \mathrm{C}$, por su parte está fuertemente influenciada por el Océano Pacífico, lo que disminuye las variaciones a través

1 Urresty, C. 2010. Geología y patrimonio geológico del Parque Nacional Bernardo O'Higgins. Informe de Avance 1 del Proyecto Innova CORFO 08CTU01-20 "Caracterización territorial del PN Bernardo O'Higgins: su potencial económico, turístico, científico y cultural”. 30 pp. del año, determinando una fluctuación estacional de las temperaturas atenuadas, comparada con el régimen de estaciones ubicadas más al interior del continente (Carrasco et al. 2002).

La cobertura vegetacional de Isla Kalau está dominada por formaciones de turberas (70\%), seguida por pastizales (5\%), y bosques costeros (25\%). En el caso de las turberas la composición florística presenta una alta representación de especies pulvinadas y ciperáceas tales como Astelia pumila, Donatia fascicularis y Schoenus antarcticus. En los pastizales costeros dominan Carex darwinii y Anthoxanthum redolens, mientras que los bosques costeros forman franjas muy densas dominadas por Nothofagus betuloides y Podocarpus nubigena.

Se realizaron muestreos y colecciones florísticas en el verano de 2009. El material recolectado fue identificado a nivel específico e infraespecífico a través del uso de floras y monografías (Correa 1969-1998, Marticorena \& Quezada 1985, Moore 1983, Matthei 1995, Marticorena \& Rodríguez 1995, Villagrán \& Barrera 2002, Novoa et al. 2006 Zuloaga et al. 2011). Para la determinación de la sinonimia y distribución de cada especie se utilizaron las bases de datos del Instituto de Botánica Darwinion (http:// www2.darwin.edu.ar/) y Plant Names (http:/www. ipni.org/). Los comentarios sobre abundancia, están basados en las estimaciones de terreno hechas por los autores utilizando el método fitosociológico de Braum - Blanquet (Mueller-Dombois \& Ellemberg, 1974).

Los ejemplares identificados fueron clasificados según su origen geográfico (Matthei 1995, Henríquez et al. 1995, Espinoza 1996, Domínguez 2007, Zuloaga et al. 2011). Para analizar aspectos dinámicos de la vegetación se determinaron los tipos biológicos propuestos por Raunkiaer (1934) y modificadas por Mueller-Dombois \& Ellemberg (1974), también se indica los usos de las plantas dados por la etnia kawésqar (Domínguez, 2010, Aguilera \& Tonko, 2011). Se identificó el estado de conservación de las especies de acuerdo con su categoría (Benoit, 1989, Baeza et al. 1998, Walter \& Gillett, 1998, Hechenleitner et al. 2005, Rodríguez et al. 2008, Rodríguez et al. 2009). Las plantas recolectadas fueron herborizadas y están depositadas en el herbario de la Universidad de Concepción (CONC). 


\section{RESULTADOS Y DISCUSIÓN}

\author{
Catálogo Florístico Comentado
}

PTERIDOPHYTA

\section{FILICOPSIDA}

\section{ASPLENIACEAE}

\section{Asplenium dareoides Desv.}

Hierba perenne, epífita. Endémica de Argentina y Chile. En Chile se distribuye entre las regiones de Coquimbo y de Magallanes. Abundante, crece en el bosque costero de Nothofagus betuloides y Podocarpus nubigena.

\section{BLECHNACEAE}

\section{Blechnum magellanicum (Desv.) Mett.}

Hierba perenne. Habita en Argentina, Islas Malvinas y Chile. En Chile se distribuye entre las regiones del Maule y de Magallanes. Escasa, crece en el borde del bosque costero de Nothofagus betuloides y Podocarpus nubigena. Conocido como xaána entre los kawésqar, se registran varios usos, el más común es como colchón dentro del at (vivienda temporal) para lo cual se utilizan sus hojas que sirven de aislante del suelo húmedo. Servía asimismo como señal para ubicarse en el bosque, como lo atestigua este texto:

Árka kukté álowep ksep tapkeráu kte ak'uás halí álowe-terrék tapkeráu čečáu-ksep-kepásna k’ejehák-hójok sa; čečáu-ksepkepásna tawesána ka kuos xaána-s aqálkče.

Hacia el interior del monte la vegetación se hace rala, dentro de él es pelado y uno se puede perder caminando siempre; solía suceder, para no perderse se quebraban [ramas] de Blechnum magellanicum. (Aguilera \& Tonko 2009: 67 § 236-7)2.

El procedimiento de señalización es descrito como sigue:

Kčéqe kse ak'uá xaána árkap ak'iéfkiar-s wa hali-c'éwe-terrék tæel... awél-ketæél ačál-s kuo kčepčé sa tarió aksék, ka aqálkče sos ku-aksék kuos jesá kuos at at k'uák'iak aksér ksep-k'uak'ákna kuos.

Aguilera, O. \& J. Tonko 2009. Isla Kalau y Territorio Adyacente. Geografía Kawésqar. CONADI XII Región y FIDE XII. Punta Arenas.
Jeqolókna asós kuos ksepahák-jewá-hójok. Xaána kčéqe kse aqálkče-erk'éna čečáu-ksepkepásna-k'ejehák-s kúka.

Kar halí álowe-terrék k'élaf táwon atál kuktép kuteké árka kupép terrék kuteké árka ko átal peterrek čečáu-ksep-kepásna čečáu-ksepsé tawesána ka kuos aqálkče-k'ejehák-hójok xaána, tarió ksep.

Aqál so jeftó so ja aksép pa kuos hólop asák asás kuos kses at hápar ja-s kuos ja-s xaána qálkče.

Las hojas de las ramas de Blechnum magellanicum, que son blancas en la parte de arriba y hacia abajo, se las dejaba [en el suelo] para señalar el camino, y habiéndolos dejado quebrados, así se podían ver y [eso indicaba] que el campamento estaba cerca y nos acercábamos.

Habiéndolas encontrado, se seguía caminando. Las hojas de ramas de Blechnum magellanicum se dejaban quebradas al ver que constantemente uno se perdía.

Los árboles de la copa hacia abajo se asemejan a un cuello en ese lugar y también arriba y más arriba también uno se podía perder y para que no pasara eso, al caminar siempre se quebraban Blechnum magellanicum, justo en el camino.

Una vez que se encuentran [las ramas] quebradas tiradas en el suelo, se sigue el camino hasta abajo derechito al campamento, hasta ahí llega el Blechnum magellanicum quebrado. (ibid: § 257-64).

El tronco del Blechnum magellanicum era empleado asimismo como juguete por los kawésqar y aparece atestiguado también como topónimo para señalar el punto donde se jugaba:

At árka kstai akér ra xaána k'iot kenčákiar jepátal-ho ak'uás taqás-ho ačáal hójok kuo ak'uás kiarahák-er-hójok sa Xaána-taqás-ho-jerás.

Ku jerás askét álowep jelái-s kok kenčahák taqahák-er-hójok ās hoo xaána k’iot asó, kenákar ak'uás aqáqar.

Kius kse so kenčáqas čerféqas; kčepqáqar kuosó eihiáqans ttaqása-k'enák jewá hójok sa kuosó kīaskuosk'ák jepátal, kuosá afsék ka kuteké awok'éna jenák ačáal-s kuok.

Kuo jeftáwos kuosá lowó kek'iák ka lowó ak'uás jeksólok jetesektálæer kteo jetəél-erk'éna asós jetétal jenák-hójok-s æes jerk iápar tawaisélok ku jerás.

Sálta kst'áksak palo sa kuos jewajókar jetának kius jeftowæéskar; qasápaks ku arp ček'éna feičék kekiá k'uas sálta kek'ia'k, ku táwon fkiar-k'ejehák- 
er-atál jorrók-s kúka kuos askét ... táuk-s taqásak at kstaip jerféjo at afčár tqalp taqástal-k'enák ačáal.

El sector de más arriba del at (vivienda temporal) es un lugar que sirve para jugar con troncos de Blechnum magellanicum cortado, por ahí lo hacían rodar, por eso lo llamaban Xaánataqás-ho-jerás (Lugar de terreno en pendiente donde se hacen rodar troncos de Blechnum magellanicum).

En esa pendiente, con mi poco conocimiento [de niña pequeña] veía cómo los cortaban y hacían rodar los troncos de Blechnum magellanicum, les cortaban y les sacaban las ramas.

Sus hojas se cortaban por completo, haciéndolo minuciosamente; se amontonaban [los tronquitos], después los empujaban [por la pendiente] $y$ rodaban, de esta manera se jugaba, cuando estaban ahí acampados.

Los ensartaban [los tronquitos de Blechnum magelanicum] como lobos de juguete, pues así lo hacían los finados a los lobos [verdaderos] cuando andaban [cazando], y lo hacía [jugando] mi difunto hermano en esa pendiente.

Confeccionaba una lanza y fabricaba un arpón de palo para ensartarlo; le ponía soga a la lanza de juguete y a la red de juguete, con ella lo arponeaba y cuando erraba, rodaba solo y se metía por la entrada del at y llegaba rodando dentro de la fogata. (ibid. $\S \S 547-56)$.

También en un cuento el narrador habla sobre la creencia de que el Blechnum magellanicum servía para establecer la duración del invierno, cuándo las noches son más largas, de acuerdo a la combustión de la planta:

Xaána sa afčártqal hápar aqápe halíkte-s kok ker-há-k'ejeháker-hójok eik'osekčéjer-hójok k’ak. Ak'éwe kstzés ak'uás kuteké aswál ói káuks kuo jekčél-s aselájer-s kuo.

Kuosá kuos xaána awás ka kuteké kius kse so awokst'atæélna. Kst'átæel awóčes erwér-aqás ói jeksór ka kuos aswál kstás ak'uás ak'éwe kstaés aselájer-s kuo.

"C'appac'éwel qal-aqás-jeké k'élok kst'atálna askét... afčár ark kupék kió-eihén kupék," aselájer-s kuo, aswál ak'uás aqápe halíkte táu kuorrá-qeikuor," æesk'ák, kuosá kius halé har kte ak'uá aselái eik'olájer-s kuo.
Kius askét.. aswál ka kuteké aqápe jekčél-s jejéskar. Kuteké aswáltqal kelentário wa jemmá jetesekčéjer-s kuosk'á.

Se echaba también una rama de Blechnum magellanicum a la fogata en la mitad del invierno, se cuenta. Por eso la noche es larga y se demora en llegar el día, lo comprobaban, se dice.

Yellos quemaban el Blechnum magellanicum $y$ las hojas quedaban hechas brasas. Al quedar sólo brasas y al ver que las brasas del helecho se demoraban en caer, entonces decían que el día era largo, [es decir,] la noche larga.

"Si se encuentra intacto [el Blechnum magellanicum en medio de la fogata] y no se esparció [la ceniza] y sólo han quedado brasas en la fogata emitiendo llamaradas," se cuenta, "entonces el día ha de ser como en la mitad del invierno," así [decía quien lo contaba] y decían y contaban que estaban en la mitad [del invierno].

Ese era su eh... [instrumento] para verificar el día y a la vez era verificador de invierno. Y lera como] el calendario que usan los hombres blancos en pleno día, era parecido a eso.

(Aguilera \& Tonko, 2010: Cuento del čénerk, §§ $31-38)^{3}$.

\section{Blechnum penna-marina (Poiret) Kuhn}

Hierba perenne. Habita en Australia, islas de los océanos Pacífico, Atlántico sur e Índico en Brasil, Bolivia, Argentina, Islas Malvinas y Chile. En Chile se distribuye entre las regiones de la Araucanía y de Magallanes. Frecuente, crece en el sotobosque del bosque de Nothofagus betuloides y en el pastizal costero. El Blechnum penna-marina se denomina nesnérwa entre los kawésqar. También puede utilizarse como colchón. No tenemos información de otro uso de esta planta, probablemente lo tenía en la antigüedad, puesto que los kawésqar distinguen las dos especies de Blechnum y les dan nombres distintos. La medicina tradicional kawésqar se perdió hacia la década de los 40, tras la instalación de la base de la Fuerza Aérea en Puerto Edén, cuando comenzaron a recibir medicinas occidentales.

3 Aguilera, O. \& J. Tonko 2010. Cuento del čénerk. T-PE140407=3. Documento de pre-publicación. Proyecto FONDECYT No. 1100334. 13 pp. 


\section{GLEICHENIACEAE}

\section{Sticherus quadripartitus (Poir.) Ching}

Hierba perenne. Endémica de Argentina y Chile. En Chile se distribuye entre las regiones de Bío-Bío y de Magallanes, también ha sido reportada en el Archipiélago de Juan Fernández. Frecuente, crece como sotobosque en el bosque costero de Nothofagus betuloides y Podocarpus nubigena.

\section{GRAMMITIDACEAE}

5. Grammitis magellanica Desv.

Hierba perenne, epífita. Habita en Nueva Zelandia, Tristan da Cunha, Sudáfrica Argentina y Chile. En Chile se distribuye entre las regiones de la Araucanía y de Magallanes, incluyendo también el Archipiélago de Juan Fernández e islas Malvinas. Escasa, crece en sectores húmedos y sombríos sobre troncos de Nothofagus betuloides. Estado de conservación: Vulnerable (Baeza et al. 1998, Rodríguez et al. 2008, Rodríguez et al. 2009).

\section{HYMENOPHYLLACEAE}

\section{Hymenoglossum cruentum (Cav.) C. Presl}

Hierba perenne, epífita. Habita en Argentina y Chile. En Chile se distribuye entre las regiones del Maule y de Magallanes, también ha sido reportada en el Archipiélago de Juan Fernández. Escasa, crece en sectores húmedos y sombríos sobre troncos de Nothofagus betuloides.

\section{Hymenophyllum cuneatum Kunze}

Hierba perenne, epífita. Endémica de Argentina y Chile. En Chile se distribuye entre las regiones de Bío-Bío y de Magallanes, también ha sido reportada en el Archipiélago de Juan Fernández. Escasa, crece en áreas sombrías sobre troncos de árboles. Estado de conservación: Vulnerable (Baeza et al. 1998, Rodríguez et al. 2008, Rodríguez et al. 2009).

\section{Hymenophyllum ferrugineum Colla}

Hierba perenne, epífita. Habita en Nueva Zelandia, Argentina y Chile. En Chile se distribuye entre las regiones de Los Ríos y de Magallanes, también ha sido reportada en el Archipiélago de Juan Fernández. Frecuente, crece bajo el dosel en el bosque costero de Nothofagus betuloides.

\section{Hymenophyllum pectinatum Cav.}

Hierba perenne, epífita. Habita en Argentina y Chile. En Chile se distribuye entre las regiones del Maule y de Magallanes, también ha sido reportada en el Archipiélago de Juan Fernández. Abundante, crece en troncos de árboles y bajo el dosel en el bosque costero en bosque costero de Nothofagus betuloides y Podocarpus nubigena. Estado de conservación: Vulnerable (Baeza et al. 1998, Rodríguez et al. 2008, Rodríguez et al. 2009).

\section{Hymenophyllum secundum Hook. et Grev.}

Hierba perenne, epífita. Endémica de Argentina y Chile. Se distribuye en Chile entre las regiones de Los Lagos y de Magallanes, también ha sido reportada en el Archipiélago de Juan Fernández. Abundante, crece bajo el dosel en el bosque de Nothofagus betuloides. Estado de conservación: Vulnerable (Baeza et al. 1998, Rodríguez et al. 2008, Rodríguez et al. 2009).

\section{Hymenophyllum tortuosum Hook. et Grev.}

Hierba perenne, epífita. Endémica de Argentina y Chile. Se distribuye en Chile entre las regiones de Los Lagos y de Magallanes, también ha sido reportada en el Archipiélago de Juan Fernández. Frecuente, crece en el interior del bosque costero de Nothofagus betuloides. Estado de conservación: Vulnerable (Baeza et al. 1998, Rodríguez et al. 2008, Rodríguez et al. 2009).

\section{Serpyllopsis caespitosa (Gaudich.) C.Chr}

Hierba perenne, epífita. Endémica de Argentina y Chile. En Chile se distribuye entre las regiones de Los Ríos y de Magallanes. Escasa, crece sobre troncos en bosque costero de Nothofagus betuloides y Podocarpus nubigena. Estado de conservación: Insuficientemente conocida (Baeza et al. 1998, Rodríguez et al. 2008, Rodríguez et al. 2009).

\section{SCHIZEACEAE}

13. Schizaea fistulosa Labill.

Hierba perenne. Habita en Australia, Nueva Zelandia, Nueva Caledonia, Malasia, Islas Malvinas, Argentina y Chile. En Chile se distribuye entre las regiones de Los Lagos y de Magallanes. Escasa, crece turberas con plantas pulvinadas y ciperáceas. Estado de conservación: Vulnerable (Baeza et al. 1998, Rodríguez et al. 2008, Rodríguez et al. 2009). 


\section{GYMNOSPERMAE}

\section{PINOPSIDA}

\section{CUPRESSACEAE}

\section{Lepidothamnus fonkii Phil.}

Subarbusto siempreverde. Endémica de Argentina y Chile. Se distribuye en Chile entre las regiones de Los Lagos y de Magallanes. Abundante, crece en una turbera ciperáceas con plantas pulvinadas. Estado de conservación: Vulnerable (Walters \& Guillett, 1998, Rodríguez et al. 2008).

\section{Podocarpus nubigena Lindl.}

Árbol siempreverde. Endémico de Argentina y Chile. En Chile se distribuye entre las regiones de la Araucanía y de Magallanes. Abundante, crece formando parte del bosque costero de Nothofagus betuloides. Estado de conservación: Vulnerable (Walter \& Gillet, 1998, Rodríguez et al. 2008).

\section{MAGNOLIOPHYTA}

\section{MAGNOLIOPSIDA}

\section{APIACEAE}

\section{Apium prostatum Labill}

Hierba perenne. Habita en Brasil, Uruguay, Argentina y Chile. En Chile se distribuye entre las regiones de Antofagasta y de Magallanes, incluyendo también el archipiélago de Juan Fernández e isla de Pascua. Frecuente en el pastizal costero, crece junto a Carex darwinii y Anthoxanthum redolens. El apio lo denominan ámtak y lo usan como comida, pero al parecer este uso es por transmisión de los chilotes, ya que lo emplean en sopas, que no era un plato tradicional.

\section{ARALIACEAE}

\section{Raukaua laetevirens (Gay) Frodin}

Árbol siempreverde. Endémico de Argentina y Chile. Se distribuye entre las regiones del Maule y de Magallanes. Frecuente, crece en el bosque costero junto a Nothofagus betuloides y Podocarpus nubigena.

\section{ASTERACEAE}

\section{Abrotanella trichoachaenia Cabrera}

Hierba perenne. Endémica de Argentina y Chile. Se distribuye entre las regiones de Aysén y de
Magallanes. Frecuente, crece entre rocas en turbera de ciperáceas y de plantas pulvinadas.

\section{Symphyotrichum sp.}

Hierba perenne. Escasa formando pequeños parches, crece en pastizal costero junto con Carex darwinii y Anthoxanthum redolens.

\section{Baccharis patagonica Hook. \& Arn.}

Arbusto erguido siempreverde. Endémico de Argentina y Chile. En Chile se distribuye entre las regiones de Bío-Bío y de Magallanes, incluyendo el Archipiélago de Juan Fernández. Escaso, crece en el ecotono entre la turbera de ciperáceas y el bosque costero de Nothofagus betuloides.

\section{Leptinella scariosa Cass.}

Hierba perenne. Habita en Argentina, Islas Malvinas y Chile. En Chile se distribuye entre las regiones de la Araucanía y de Magallanes. Escasa, en turbera de ciperáceas con plantas pulvinadas.

22. Senecio acanthifolius Hombr. et Jacquinot

Hierba perenne. Endémica de Argentina y Chile. Se distribuye entre las regiones de Los Lagos y de Magallanes. Frecuente, crece en el pastizal costero junto a Carex darwinii y Anthoxanthum redolens.

\section{Senecio smithii DC.}

Hierba perenne. Endémica de Argentina y Chile. En Chile se distribuye entre las regiones de la Araucanía y de Magallanes. Frecuente en la desembocadura de ríos y zonas pantanosas, crece junto a Carex darwinii y Anthoxanthum redolens. Los kawésqar lo denominan jeqáqa, su uso medicinal era para curar heridas, se calentaba al fuego y su jugo se vertía sobre la herida, y luego se cubría ésta con una cataplasma hecha con las hojas calentadas al fuego, además se usaba como tratamiento para la sarna; con el tubérculo jugaban los niños varones, servía de blanco para flechas.

\section{Senecio trifurcatus (G.Forst.) Less.}

Hierba perenne. Endémica de Argentina y Chile. En Chile se distribuye entre las regiones de la Araucanía y de Magallanes. Escasa, crece en turbera de ciperáceas con plantas pulvinadas. Los kawésqar lo denominan jeqáqa, su uso medicinal era para curar heridas, se calentaba al fuego y su jugo 
se vertía sobre la herida, y luego se cubría ésta con una cataplasma hecha con las hojas calentadas al fuego, además se usaba como tratamiento para la sarna; con el tubérculo jugaban los niños varones, servía de blanco para flechas.

\section{BERBERIDACEAE}

25. Berberis ilicifolia L.f.

Arbusto siempreverde. Endémico de Argentina y Chile. En Chile se distribuye entre las regiones de Los Lagos y de Magallanes. Escaso, crece al interior del bosque costero de Nothofagus betuloides y Podocarpus nubigena.

26. Berberis microphylla G. Forst.

Arbusto siempreverde. Endémico de Argentina y Chile. En Chile se distribuye entre las regiones de O'Higgins y de Magallanes. Frecuente, crece en sitios abiertos en bosque costero.

Los frutos de ambas especies de Berberis son recolectados y consumidos por los kawésqar. Sus nombres son čaulájek y wajamáskar, respectivamente.

\section{BIGNONIACEAE}

\section{Campsidium valdivianum (Phil.) Skottsb.}

Enredadera perenne. Endémica de Argentina y Chile. En Chile se distribuye entre las regiones de la Araucanía y de Magallanes. Abundante, crece en el bosque costero trepando los troncos de Nothofagus betuloides. Enredadera perenne. Endémica de Argentina y Chile. En Chile se distribuye entre las regiones de la Araucanía y de Magallanes. Abundante, crece en el bosque costero trepando los troncos de Nothofagus betuloides. Los kawésqar lo llaman ksqawá y se usaba para hacer lazos para cazar pájaros; para las costuras y asas de los cestos, lo usaban como cordón para cerrar una bolsa de cuero de lobo marino que servía para conservar grasa de lobo que se dejaba enterrada en turberas; también se empleaba en la construcción de las antiguas canoas de corteza:

Ksqawá kar ar atzél kuteké wəés-tqal erjá, c'apasjetána aqapés-kar sa kuosk'ák kuteké tái kajésqa lejés-kar sa.

Campsidium valdivianum se encuentra en los árboles y también crece en tierra en el suelo, sirve para coser canastos también y lazo para cazar pájaros (Aguilera 2006: T-PE-300184B=58).

\section{BRASSICACEAE}

28. Cardamine glacialis (G. Forst.) DC.

Hierba perenne. Endémico Argentina y Chile.

En Chile se distribuye entre las regiones de Los Lagos y de Magallanes. Escasa, crece en el pastizal costero de Carex darwinii y Anthoxanthum redolens.

\section{CAMPANULACEAE}

29. Lobelia sp.

Hierba perenne. Habita en Argentina, Islas Malvinas y Chile. Se distribuye en todo Chile. Escasa, crece en el pastizal costero, en parches junto a Carex darwinii y Anthoxanthum redolens.

\section{CARYOPHYLLACEAE}

30. Colobanthus quitensis (Kunth) Bartling

Hierba perenne. Habita en Bolivia, Argentina, Islas Malvinas y Chile. Se distribuye en todo Chile, incluyendo la península Antártica. Escasa en pastizal costero, crece entre rocas formando pequeños cojines.

\section{Stellaria pallida (Dumort.) Piré}

Hierba perenne introducida. Originaria de Europa. En Chile ha sido reportada para Región Metropolitana de Santiago y para Magallanes. Frecuente, crece en el pastizal costero.

\section{CELASTRACEAE}

32. Maytenus magellanica (Lam.) Hook.f.

Árbol siempreverde. Endémico de Argentina y Chile. En Chile se distribuye entre las regiones del Maule y de Magallanes. Frecuente, crece en sitios abiertos en el bosque costero de Nothofagus betuloides y Podocarpus nubigena. Los kawésqar sólo lo utilizan como leña y lo denominan ac'érkians:

Ac'érkians sa afcár-s, afcár sa ac'érkians ac'érkians ánkcók ka kuteké às.

El Maytenus magellanica es leña, leña es el M. magellanica seco (Aguilera, 2006: T-PE-210184=20) ${ }^{4}$.

\section{CORNACEAE}

33. Griselinia ruscifolia (Clos) Ball

Enredadera perenne. Endémica Argentina y Chile. En Chile se distribuye entre las regiones de Los Lagos y de Magallanes. Frecuente, crece en

Aguilera, O. 2006. Corpus de textos archivo sonoro 19751985.MS 
sitios sombríos en el bosque costero de Nothofagus betuloides y Podocarpus nubigena.

\section{CRASSULACEAE}

34. Crassula moschata G.Forst.

Hierba anual. Habita en Argentina, Islas Malvinas y Chile. Se distribuye entre las regiones de los Lagos y de Magallanes. Abundante, crece formando pequeños céspedes en el pastizal costero de Carex darwinii y Anthoxanthum redolens.

\section{DESFONTAINIACEAE}

\section{Desfontainia fulgens D.Don}

Arbusto perenne. Habita en Bolivia, Argentina y Chile. En Chile se distribuye entre las regiones del Maule y de Magallanes. Abundante, crece en los claros del bosque costero de Nothofagus betuloides y Podocarpus nubigena. El nombre kawésqar es qaléskar. Las ramas y tronco se utilizan como leña, sus hojas sirven para infusión en reemplazo de té o café, es un uso moderno, ya que en la antigüedad los kawésqar no consumían este tipo de bebidas.

qalésxar sa æerjá-atál páu æes-kápar ja-atál, afčár sa afčár kenčé-sekcé-s kuos kse akstápkta-af sa.

El Desfontainea fulgens crece afuera aquí cerca hay, es leña, se corta y su hoja es pinchadora (Aguilera 2006: T-PE-300184B=55).

Qaléskar kefé c’afaséktal, kius kse-s awókan-s məelmás c'afaséktal os kefé.

El Desfontainea fulgens se bebe como café, sus hojas se cuecen, se hierven y se bebe como café (Aguilera 2006: T-PE-210184=13).

\section{DONATIACEAE}

36. Donatia fascicularis J.R. Forst. \& G. Forst.

Subarbusto perenne en cojín. Endémico de Argentina y Chile. En Chile se distribuye entre las regiones de Bío-Bío hasta Magallanes. Abundante, crece en turberas de ciperáceas y de plantas pulvinadas, las que se ubican en el centro de la isla.

\section{DROSERACEAE}

37. Drosera uniflora Willd.

Hierba perenne, insectívora, con pelos glandulares que le confieren un color rojizo. Habita en Argentina, Islas Malvinas y Chile. En Chile se distribuye entre las regiones de Bío-Bío y de Magallanes. Abundante, crece en las turberas de ciperáceas y de plantas pulvinadas.

\section{EMPETRACEAE}

38. Empetrum rubrum Vahl ex Willd.

Subarbusto bajo o rastrero. Habita en Argentina, Islas Malvinas y Chile. En Chile se distribuye entre las regiones de Valparaíso y de Magallanes, también ha sido reportada en el Archipiélago de Juan Fernández. Escaso en turberas de ciperáceas y de plantas pulvinadas, crece entre rocas que sobresalen en la turbera. Su nombre kawésqar es ačáksa, el fruto es apetecido y se recolectaba en las cercanías de los campamentos temporales. Hoy también se lo recolecta, se consume fresco o preparan mermeladas.

Ačáksa sa har æes-kar qálak je-akstá-ar, jekt'osekčéjer ās ačáksa jec'ói-s, asáqe-s wa kupép.

Empetrum rubrum habrá aquí en la turbera, se coge con la mano el fruto del Empetrum rubrum, es comida también (Aguilera 2006: T-PE-210184=22).

\section{EPACRIDACEAE}

\section{Lebetanthus myrsinites (Lam.) Dusén}

Subarbusto trepador. Endémico de Argentina y Chile. En Chile se distribuye entre las regiones de Los Lagos y de Magallanes. Frecuente, crece sobre troncos en el bosque costero de coigüe de Magallanes y mañio.

\section{ERICACEAE}

40. Gaultheria antarctica Hook.f.

Subarbusto siempreverde. Endémico de Argentina y Chile. En Chile se distribuye entre las regiones de la Araucanía y de Magallanes. Escasa, crece en turberas de ciperáceas y plantas pulvinadas.

\section{Gaultheria mucronata (L. f.) Hook. \& Arn.}

Subarbusto siempreverde. Endémico de Argentina y Chile. En Chile se distribuye entre las regiones del Los Lagos y de Magallanes. Abundante, crece en sitios abiertos en el bosque costero de Nothofagus betuloides y Podocarpus nubigena. Los kawésqar recolectaban y consumían sus frutos. Su nombre en kawésqar es ačás.

\section{Gaultheria pumila (L. f.) D.J. Middleton}

Subarbusto siempreverde. Habita en Argentina, Islas Malvinas y Chile. En Chile se distribuyen desde Aysén hasta Magallanes. Es frecuente, crece en turberas de ciperáceas y de plantas pulvinadas. Los kawésqar recolectaban y consumían sus frutos. Su nombre en kawésqar es ačás. 
Ačás wa æerjá árka qálak kuos ačás ačétqa-k'éja-atál ās, kejéjo-jeké kua kuos asáqe sa k’ak.

La chaura crece en la turbera arriba y la chaura madura, es rojita y también se come (Aguilera 2006: T-PE-300184B=49).

\section{ESCALLONIACEAE}

43. Escallonia serrata Sm.

Arbusto siempreverde. Endémico de Argentina y Chile. En Chile se distribuye entre las regiones de Los Lagos y de Magallanes. Escasa, crece en el borde del bosque costero de Nothofagus betuloides y Podocarpus nubigena.

\section{GESNERIACEAE}

44. Mitraria coccinea Cav.

Hierba perenne, epífita. Endémica de Argentina y Chile. En Chile se distribuye entre las regiones de Coquimbo y de Magallanes. Escasa, crece en el borde del bosque costero de Nothofagus betuloides y Podocarpus nubigena.

\section{GUNNERACEAE}

45. Gunnera lobata Hook. f.

Hierba perenne. Endémica de Argentina y Chile. En Chile se distribuye entre las regiones de Los Lagos y de Magallanes. Frecuente en turberas. Frecuente, crece en turberas de Schoenus antarcticus y Astelia pumila.

\section{Gunnera magellanica Lam.}

Hierba perenne. Endémica de Argentina y Chile. En Chile se distribuye entre las regiones $\mathrm{Me}$ tropolitana y de Magallanes. Abundante, crece en el pastizal costero de Carex darwinii y Anthoxanthum redolens.

\section{LENTIBULARIACEAE}

\section{Pinguicula antarctica Vahl}

Hierba perenne. Endémica de Argentina y Chile. En Chile se distribuye entre las regiones de la Araucanía y de Magallanes. Escasa, crece en turberas de ciperáceas y de plantas pulvinadas.

\section{MISODENDRACEAE}

48. Misodendrum punctulatum Banks ex DC.

Planta hemiparásita. Endémica de Argentina y Chile. En Chile se distribuye entre las regiones del Maule y de Magallanes. Frecuente, crece sobre coigües de Magallanes en turbera de ciperáceas y de plantas pulvinadas.

\section{MYRTACEAE}

49. Myrteola nummularia (Poir.) O. Berg

Subarbusto rastrero. Habita en Bolivia, Argentina, Islas Malvinas y Chile. En Chile se distribuye entre las regiones de Bío-Bío y de Magallanes. Frecuente, crece en las turberas de ciperáceas con plantas pulvinadas. Se caracteriza por su fragancia. Los kawésqar recolectaban y comían sus frutos (Gabriela Paterito año 2008, com. pers.).

50. Tepualia stipularis (Hook. \& Arn.) Griseb.

Arbusto. Endémico de Argentina y Chile. En Chile se distribuye entre las regiones del Maule y de Magallanes. Abundante, crece en el interior del bosque costero de Nothofagus betuloides y Podocarpus nubigena. Su nombre kawésqar es k'iesk'iáluxar y su uso principal es para leña:

k'iesk'iáluxar k'oának arkápe kuos wæes jak'etæél-atál, afcár sa afcár-s kuos kenceséktal co as, ánnas sa ártqa-ketæél as, jekstás-kte sa ártqa, aqápe sa kius ártqa k'élok, jekstás-kte kuos jaqás-terrék kius ær-ketálap.

El Tepualia stipularis se encuentra en el bosque, en todas partes hay, es leña y se lo corta, crece, crece, en primavera crece, en invierno no crece, en primavera y verano crece (Aguilera 2006: T-PE-200184A=9).

\section{NOTHOFAGACEAE}

51. Nothofagus antarctica (G. Forst.) Oerst.

Árbol caducifolio. Endémico de Argentina y Chile. En Chile se distribuye entre las regiones del Maule y de Magallanes. Escasa, crece en turberas de ciperáceas con plantas pulvinadas.

\section{Nothofagus betuloides (Mirb.) Oerst.}

Árbol siempreverde. Endémico Argentina y Chile. En Chile se distribuye entre las regiones de Los Lagos y de Magallanes. Dominante en bosque siempreverde costero. Su nombre kawésqar es jerkiánap, se utiliza como leña y antiguamente para fabricación de canoas y en la construcción de la vivienda temporal (at), tanto ramas como varas: Jerkiánap sa kuoskás arrakstáwar, hoik'épqar kuteké kius ánčok-s wa kuos afčár awesekčé-s. 
El Nothofagus betuloides igual es grande, grueso y seco es para leña y se quema (Aguilera 2006: T-PE-210184_6).

coigüe-s wa atáksta sewéjep ak'uás jetesekčéhjok-qe-ās kuos; jeksólok wa kuos kenčéqal kenčéksta ak’uás kuteké kájef kečelájer-s kok atalóna-kesekčéjerhójok kuo-qei-so-kuar kuos. Kiáu wa atáksta káriek-s kok ker kuo t’ak'iéksta-tálaf-s kóka ataséna kenčaséna paks ačáal hójok. Kenčéksta sewéjep-ker ka kuos atáksta sewéjep. Kius atás so c’áfsas pe tawónak'éjes-hójok sa coigüe kius čelejés kuos kájef atálai kenčelái-s kok.

El Nothofagus betuloides es difícil de partir; el finado lo derribaba era difícil de trozar y también cuando se fabricaban canoas con esta madera siempre era muy difícil de labrar. A veces era fácil de cortar y de trabajar la canoa. Cuando es difícil de trozar también es difícil de labrar. Cuando se labra para hacer canoas el nudo del coigüe está a la vista

(Aguilera \&Tonko 2006: T.SA-101106=1c-1 $\S 80-84)$.

\section{ONAGRACEAE}

53. Fuchsia magellanica Lam.

Arbusto. Habita en Bolivia, Argentina y Chile. En Chile se distribuye entre las regiones de Valparaíso y de Magallanes. Frecuente, crece en sitios abiertos en el bosque costero de Nothofagus betuloides y Podocarpus nubigena. Su nombre kawésqar es qawarákar; por lo general los niños chupan el néctar de las flores de F. magellanica por su dulzor, se desconoce otro uso (Gabriela Paterito año 2008, com. pers.).

\section{PLANTAGINACEAE}

54. Hebe elliptica (G. Forst.) Pennell

Arbusto perenne. Habita en Argentina, Islas Malvinas y Chile. En Chile se distribuye entre las regiones de Aysén y de Magallanes. Frecuente, crece en el pastizal costero y en el interior del bosque costero de Nothofagus betuloides y Podocarpus nubigena. Su nombre kawésqar es áit'æl, abundante en la costa exterior (málte). Lo usan de combustible, para incrementar una fogata o al encender el fuego:

Kuosá karkarra ak'uás kius áit'æel sa málte kčéqe-s ja ketaél-s sa áitæl táusa ka kuteké karkárra. Kius k'ies sa jet'ápkamána kuos ja ketaél-hójok parragao, k’iesqámai ka kuteké k’ies wæes kúkta k'élok jautáuk ka k’iesqámai sa jáu ketaél sa málte sa k’iesqámai kiáwel.

Es pelado, los hebe son las únicas ramas que se encuentran en la costa del mar exterior, solamente hebe y es pelado. Su bosque es tupido y parejo, se encuentra ahí aparragado, selvático y como lugar boscoso no es, en la costa interior hay bosque selvático, en la costa exterior no hay bosque selvático (Aguilera \& Tonko 2009: § 614-16).

Kius awetqána-ho sa at páu pe trelk kuos kiut ait'æél atæél-kejér c'éwe ankčók ja-s ka ait'æél paks ja kuo aqál-kerhá awó-kerhá awókče-k’enák-hójok kupép.

El lugar en donde se hacían la fogata provisoria se encontraba del campamento más afuera, es un sector boscoso allñi se encuentran bosques de hebe y algunos están secos. Los hebe que están secos los quebraban [con la rodilla o bien con la mano] y los echaban a la fogata y con eso se avivaba la fogata, siempre hacían fogatas con los hebe [en ese lugar] (Aguilera \&Tonko 2006: T-SA$190207=2$ a $§ 90)$.

\section{Plantago barbata G. Forst.}

Hierba perenne. Endémica de Chile y Argentina. En Chile se distribuye entre las regiones de Atacama y de Magallanes. Frecuente, crece en el pastizal costero.

\section{PROTEACEAE}

56. Embothrium coccineum J.R. Forst. \& G. Forst. Árbol o arbusto siempreverde. Endémica de Argentina y Chile. En Chile se distribuye entre las regiones del Maule y de Magallanes. Frecuente, crece entre rocosas que sobresalen en turbera de ciperáceas y de plantas pulvinadas. Su nombre kawésqar es jelá. Su corteza se empleaba para construir recipientes para transportar agua potable en la canoa o tenerla en la vivienda temporal (at); también se la utilizaba en la construcción de canoas. En la actualidad no tiene ningún uso:

Jelá sa jekstás kejéro, lájep-jeké erkanána, jekstás kejéro sa jelá-s lájep; jelá sa lájep-s kuteké kájef akséns-kar.

El Embothrium coccineum tiene flores rojas, es lindo crecido, las flores rojas del Embothrium coccineum son hermosas; el Embothrium coccineum es 
hermoso y sirve para hacer canoa (Aguilera 2006: T-PE-210184=10).

\section{Lomatia ferruginea (Cav.) R. Br.}

Árbol o arbusto siempreverde. Endémica de Argentina y Chile. En Chile se distribuye entre las regiones de Bío-Bío y de Magallanes. Escaso, crece en el bosque costero de Nothofagus betuloides y Podocarpus nubigena.

\section{RANUNCULACEAE}

58. Caltha appendiculata Pers.

Hierba perenne. Endémica de Argentina y Chile. En Chile se distribuye entre las regiones del Libertador Bernardo O'Higgins y de Magallanes. Escasa, crece en turbera de ciperáceas y de plantas pulvinadas.

\section{Caltha dioneifolia Hook.f.}

Hierba perenne. Endémica de Argentina y Chile. En Chile se distribuye entre las regiones de Aysén y de Magallanes. Frecuente, crece formado cojines en turberas de ciperáceas y de plantas pulvinadas en las laderas de cerros.

\section{ROSACEAE}

60. Acaena ovalifolia Ruiz et Pav.

Hierba perenne. Habita en Argentina, Islas Malvinas y Chile. En Chile se distribuye entre las regiones del Maule y de Magallanes. Escasa, crece en los pastizales costeros.

\section{RUBIACEAE}

\section{Nertera granadensis (Mutis ex L. f.) Druce}

Hierba perenne, epífita. Argentina, Islas Malvinas y Chile. En Chile se distribuye entre las regiones de la Araucanía y de Magallanes. Frecuente en el bosque costero, crece en sitios sombríos y húmedos.

\section{SAXIFRAGACEAE}

62. Chrysosplenium macranthum Hook.

Hierba perenne. Endémica de Argentina y Chile. En Chile se distribuye en la Región de Magallanes. Escasa, crece en el pastizal costero.

\section{STYLIDIACEAE}

63. Phyllachne uliginosa J.R. Forst. \& G. Forst.

Subarbusto. Endémica de Argentina y Chile. En Chile se distribuye entre las regiones de Aysén y de Magallanes. Escaso, crece en las turberas ciperáceas con plantas pulvinadas.

\section{VALERIANACEAE}

64. Valeriana leucocarpa DC.

Hierba perenne. Endémica de Argentina $y$ Chile. En Chile se distribuye entre las regiones de O'Higgins y de Magallanes. Escasa, crece en el pastizal costero.

\section{WINTERACEAE}

65. Drimys winteri J.R. Forst. \& G. Forst.

Árbol siempreverde. Endémico de Argentina y Chile. En Chile se distribuye entre las regiones de Coquimbo y de Magallanes. Frecuente. Crece en el bosque costero de Nothofagus betuloides. La especie es conocida como saltáxar entre los kawésqar, registra varios usos, como en la armazón del at (vivienda temporal), y en la antigüedad se utilizaba su corteza en el calafateo de la canoa, además de servir como varillas delgadas y rodrigones para largueros, travesaños y nervaduras de la canoa; con uso medicinal, se empleaba la corteza en lavados y aplicaciones húmedas en quemaduras y heridas. El uso para la construcción de arpones es documentado por Emperaire (1963: 180) como sigue: "La cabeza móvil del arpón para focas es fijada a un asta de dos metros de largo. La hacen con madera de canelo. En su extremo dejan una cavidad elíptica destinada a recibir la paleta de inserción. El extremo inferior del asta es hendido en todo el diámetro, ahuecado después y fuertemente ligado por medio de una delgada correa de cuero. La cuerda, que también es de cuero bruto torcido, de 20 ó 30 metros de largo, se fija a la cabeza móvil del arpón sobre el pedúnculo más o menos circular situado entre las barbaduras y la paleta de inserción, amarrada en dos sitios al asta, el resto se enrolla como un lazo. Armado así, el arpón está listo para ser empleado."

En la literatura oral, un canelo de grandes proporciones que se encuentra en uno de los brazos del Brazo Norte, un largo seno que se interna profundamente en la parte sur de la isla Wellington por el canal Trinidad, es madre de un niño que aparece al pie de dicho árbol y se transforma en uno de los héroes de los mitos kawésqar:

Kuosá saltákar k'iotc'éwe aqtál atzél asó har so kuos tælamás kius ktæélna kuos Saltákar; saltákar 
jeké ak'uás aselái kiarlájer kuosá kuos, saltákar hóik'epkar ak'uas c'ekiá... ka kuos tæel-k'enák-ket kútqal pe t’æes c'éwek kuo. Kuo hójok eik'uahák ječér-aháker kuos tæel-hójok arrakstáwar saltákar ājākstāwar halé c'éwe-terrék kawés qarqára p'árroks kawésqar čečáu-asakána kawesqá tæel-s kuos. Saltákar jeké táwon ak'uás hójok aselájer-s kuos tæel-s kuos

Ese niño estaba llorando al pie del tronco del canelo [y la gente que allí habitaba] lo tomó en sus brazos y finalmente se quedó con el nombre de Canelo; se dice, se lo designa como hijo del canelo, es un canelo grande y aún allí se encontrará en ese lugar en ese lomo y entremedio de los matorrales. Allí se encontraba, la gente lo señalaba y contaba [que ese árbol era la madre del niño], era grande el canelo era graaandeeee y abajo por la parte del tronco la corteza era un rojo claro, parecía como una persona desnuda. Se dice y se cuenta que este canelo que se encontraba ahí tuvo un hijo (Aguilera \&Tonko 2006-7: T-PE-010307=1 §§ 136-140) ${ }^{5}$.

El fruto del Drimys winteri es comestible, pero los kawésqar evitan las semillas, que señalan como picantes:

Saltáxar sa jecói táwon kuoskás, asáqe sa kius jecói-s. Asáqe sa t'al sa k'ak, kius kse sa t'al-s, t’al ka afkstái čælčæes asé; kius káwes wa t’al-s k’ak. Česékar-s wa tóu-akselái? Kius kse ka kuteké kius káwes. Kuo táwon af-kiawélna paks. Kius kse ka kuteké ken c'afá-afsár. Saltáxar sa kuos wal ka kuteké kájef éiwol kuteké jemóxar. Jemókar ječáka-s kok.

El Drimys winteri tiene fruto, es comestible su fruto. Cuando se come es picante, igual, su hoja es picante, es picante y duele la boca; su corteza es picante, igual. Sirve para remedio, ¿no? Su hoja como también su corteza. Con eso se sana rápido. Su hoja y su jugo se beben. El Drimys winteri sirve para hacer cosas, tanto bancadas de bote como remos (Aguilera 2006: T-PE-210184=11).

\section{LILIOPSIDA}

\section{ASTELIACEAE}

66. Astelia pumila (G. Forst.) Gaudich

Hierba perenne. Habita en Argentina, Islas Malvinas y Chile. Se distribuye entre las regiones

5 Aguilera, O. \& J. Tonko 2006-07. Corpus de textos archivo sonoro 2006-2007. MS del Maule y de Magallanes. Abundante, crece en turberas de ciperáceas con plantas pulvinadas junto a Donatia fascicularis.

\section{CENTROLEPIDACEAE}

67. Gaimardia australis Gaudich.

Hierba perenne Endémica de Argentina y Chile. En Chile se distribuye entre las regiones de Los Lagos y de Magallanes. Frecuente, crece en turberas de ciperáceas con plantas pulvinadas.

\section{CYPERACEAE}

68. Carex darwinii Boott

Hierba perenne. Endémica de Argentina y Chile. En Chile se distribuye entre las regiones de la Araucanía y de Magallanes. Frecuente, crece en el pastizal costero.

\section{Oreobolus obtusangulus Gaudich}

Hierba perenne. Habita en Argentina, Islas Malvinas y Chile. En Chile se distribuye entre las regiones de Bío-Bío y de Magallanes. Frecuente, crece en turberas de ciperáceas con plantas pulvinadas. Su nombre kawésqar es kuolások, antiguamente se lo usaba como estopa para las canoas:

Kuolások kuos c'afalái-jekanétqal éihen-ketzél sa, kájef, kájef apks sa kuos askét al-sekčéjer-hójo k-k'ejá-ačál-hóraras kuos kémna-ačéjer ās karáu.

El Oreobolus obtusangulus se encuentra en las lagunas, era estopa para las embarcaciones y antes se recogía, actualmente ya no se usa (Aguilera 2006: T-PE-300184B=52).

\section{Schoenus antarcticus (Hook. f.) Dusén}

Hierba perenne. Endémica de Argentina y Chile. En Chile se distribuye entre las regiones de Aysén y de Magallanes. Abundante, crece en turberas de ciperáceas con plantas pulvinadas.

\section{IRIDACEAE}

71. Tapeinia pumila (G.Forst.) Baillon

Hierba perenne. Endémica de Argentina y Chile. En Chile se distribuye entre las regiones de la Araucanía y de Magallanes. Frecuente, crece en turberas de ciperáceas con plantas pulvinadas. 


\section{JUNCACEAE}

72. Juncus balticus Willd. ssp. mexicanus (Willd. ex Roem \& Schult.) Kirschner

Hierba perenne. Habita en Argentina y Chile. En Chile se distribuye entre las regiones de Antofagasta y de Magallanes, incluyendo también el archipiélago de Juan Fernández e isla de Pascua. Escasa, crece en el pastizal costero.

\section{Marsippospermum grandiflorum (L.f.) Hook.}

Hierba perenne. Habita en Argentina, Islas Malvinas y Chile. En Chile se distribuye entre las regiones de Bío-Bío y de Magallanes. Abundante, crece en las turberas de ciperáceas con plantas pulvinadas. El nombre kawésqar es c'apás, lo empleaban principalmente para fabricar sogas y cestos de diverso uso (recolección de mariscos, para guardar cosas). Últimamente lo fabrican como souvenirs que comercian en los barcos que pasan por Puerto Edén:

Aswálak če c'apás kennæés-sekué c'apasjetána jetás-kar os, jemmásek čas-kar-qei jetepéna.

Mañana voy a salir a buscar Marsippospermum grandiflorum para hacer canastos, los haré para venderlos en el buque (Aguilera 2006: T-PE-020284A=17).

Las sogas de este material se utilizaban en la fabricación del at para atar las varas del armazón; asimismo lo trenzaban para la fabricar cabos para amarrar la canoa. Por otra parte, Emperaire (op. cit.: 222) señala un juego en el que se utilizaba esta planta: "Entre los grandes, no subsiste sino un juego tranquilo, que se practica ordinariamente en posición horizontal. Consiste en fingir que se amarra lo más rápido posible la canoa a uno de los postes de la choza y se juega por medio de juncos o de cabos de cuerda. A una señal dada, cada uno se amarra el dedo o la muñeca, que representan la canoa, a un poste de la choza. A la segunda señal, "desamarrad la canoa", se trata de deshacer rápidamente el nudo, y el que se atrasa en liberarse, pierde. El juego puede así durar horas"

\section{Rostkovia magellanica (Lam.) Hook.f.}

Hierba perenne. Habita en Argentina, Islas Malvinas y Chile. En Chile se distribuye entre las regiones de Los Lagos y de Magallanes. Frecuente, crece en las turberas de ciperáceas.

\section{PHILESIACEAE}

75. Luzuriaga marginata (Gaertn.) Benth.

Subarbusto. Habita en Argentina, Islas Malvinas y Chile. En Chile se distribuye entre la Región de Los Lagos y de Magallanes. Frecuente, crece en el margen de los bordes del bosque de coigüe.

\section{Philesia magellanica J.F.Gmel.}

Subarbusto siempreverde, trepador con vistosas flores vistosas. Endémico de Argentina y Chile. Se distribuye entre las regiones de Los Lagos y de Magallanes. Frecuente, crece en los bosques costeros y en turberas con afloramientos rocosos. Su nombre kawésqar es kelakéla y el fruto lo denominan maláute, sus flores son comestibles; Emperaire (op cit.: 176) menciona cables de Philesia magellanica usado antiguamente: "En otra época hacían, con raíces finas de copihue, un cable grueso de varias hebras de una pulgada de diámetro, que constituía un notable trabajo de trenzado. De él no queda más que el recuerdo."

\section{POACEAE}

77. Anthoxanthum redolens (Vahl) P. Royen

Hierba perenne. Habita en Argentina, Islas

Malvinas y Chile. En Chile se distribuye entre las regiones de Coquimbo y de Magallanes. Abundante, crece en el pastizal costero.

\section{Deschampsia kingii (Hook.f.) E.Desv.}

Hierba perenne. Endémica de Argentina y Chile.

En Chile se distribuye entre las regiones de Aysén y de Magallanes. Escasa, formando parte del pastizal.

\section{Festuca cirrosa (Speg.) Parodi}

Hierba perenne. Endémica de Chile y Argentina. En Chile descrita sola para Magallanes. Escasa, formando parte del pastizal costero.

\section{Libertia chilensis (Molina) Gunckel} Hierba perenne. Endémica de Argentina y Chile. En Chile se distribuye entre las regiones de Los Lagos y de Magallanes. Escasa, crece en el pastizal costero.

El catálogo de la flora vascular de la isla Kalau queda constituido por 80 especies, 67 géneros y 48 familias (Tabla I) que representan un 7\% de la biodiversidad de plantas vasculares citadas para la Región de Magallanes (Henríquez et al. 1995). Las familias más diversas son: Hymenophyllaceae 
(3 géneros/ 7 especies), Asteraceae (5 géneros/7 especies) y Poaceae (4/4). Los géneros mejor representados son: Hymenophyllum (5 especies), Senecio (3) y Gaultheria (3), 59 géneros representados por una especie. De las especies reportadas para la isla, 29 son endémicas de Chile y Argentina y 9 presentan problemas de conservación, siendo 8 especies clasificadas en categoría de Vulnerable, y 1 especie en categoría Insuficientemente conocida.
La especie introducida corresponde al $1 \% \mathrm{de}$ la flora total, para una superficie de 1.629,92 hectáreas. Una tendencia similar ha sido detectada en el Parque Nacional Cabo de Hornos con una superficie de 63.093 hectáreas, un ecosistema insular donde se registró 1,8\% de especies introducidas (Rozzi et al. 2004). El bajo porcentaje de especies introducidas, confirma el buen estado de conservación de la vegetación, quizás se pueda deber a la disminución

Tabla I. Estructura taxonómica de las plantas vasculares de la isla Kalau en el Parque Nacional Bernardo O'Higgins.

\begin{tabular}{lccc} 
Clases & Familias & Géneros & Especies $\left(\mathrm{N}^{\circ}\right)$ \\
\hline Magnoliopsida & 35 & 42 & 50 \\
Liliopsida & 7 & 15 & 15 \\
Filicopsida & 6 & 8 & 13 \\
Pinopsida & 1 & 2 & 2 \\
TOTAL & 48 & 67 & 80
\end{tabular}

de la presión ejercida en la actividad de pesca, caza y recolección de frutos ejercida en el pasado por los kawésqar, la cual hoy prácticamente no existe.

En este estudio, se reconoce el uso dado tradicionalmente por los Kawésqar a 23 especies que representa el 29\% de la flora total. Los principales usos fueron: 1) comestible con un $43 \%$ siendo muchos de estos alimentos hoy dejados de consumir perdiéndose progresivamente el conocimiento, 2)
$30 \%$ con fines de construcción como materia prima en la elaboración de viviendas, canoas, sogas, recipientes y cestas, 3) un $21 \%$ como combustible y 4) un $13 \%$ medicinal. La especie Drimys winteri es la que presenta la mayor cantidad de usos (Tabla II). Las propiedades medicinales más citadas son cicatrizantes para heridas. Es importante destacar que este catálogo se centra en la isla Kalau, no a todo el territorio ancestral.

Tabla II. Principales aplicaciones por categoría de usos encontrados de la flora por los Kawésqar, isla Kalau en el Parque Nacional Bernardo O'Higgins.

\begin{tabular}{|c|c|c|c|c|}
\hline Especies & Nombre kawésqar & Usos & Descripción & Ecosistema \\
\hline Blechnum magellanicum & xaána & Construcción & Colchón, señaletica y juegos & Bosque \\
\hline Blechnum penna-marina & nesnérwa & Construcción & Colchón & Bosque \\
\hline Apium prostatum & ámtak & Alimenticio & Fruto comestible & Pastizal \\
\hline Senecio smithii & jeqáqa & Medicinal & Curar heridas & Pastizal \\
\hline Senecio trifurcatus & jeqáqa & Medicinal & Curar heridas & Pastizal \\
\hline Berberis ilicifolia L.f. & čaulájek & Alimenticio & Fruto comestible & Bosque \\
\hline Berberis microphylla & wajamáskar & Alimenticio & Fruto comestible & Bosque \\
\hline Maytenus magellanica & ac'érkians & Combustible & Leña & Bosque \\
\hline Desfontainia fulgens & qaléskar & Combustible/infusión & Leña/hojas & Bosque \\
\hline Empetrum rubrum & ačáksa & Alimenticio & Fruto comestible & Turbera \\
\hline Gaultheria mucronata & ačás & Alimenticio & Fruto comestible & Bosque \\
\hline Gaultheria pumila & ačás & Alimenticio & Fruto comestible & Turbera \\
\hline Myrteola nummularia & & Alimenticio & Fruto comestible & Turbera \\
\hline Tepualia stipularis & k'iesk'iáluxar & Combustible & Leña & Turbera \\
\hline Fuchsia magellanica & qawarákar & Alimenticio & Fruto comestible & Bosque \\
\hline Hebe elliptica & áit'æl & Combustible & Leña & Bosque \\
\hline Embothrium coccineum & jelá & Construcción & Canoa/recipientes para el agua & Bosque \\
\hline Drimys winteri & saltáxar & $\begin{array}{l}\text { Construcción, doméstico, } \\
\text { medicinal, alimenticio }\end{array}$ & Canoa/curar heridas/fruto comestible & Bosque \\
\hline Oreobolus obtusangulus & kuolások & Construcción & Estopa para las canoas & Turbera \\
\hline Marsippospermum grandiflorum & c'apás & Construcción & Sogas, cestos y juegos & Turbera \\
\hline Philesia magellanica & kelakéla & Alimenticio & Fruto comestible & Turbera \\
\hline
\end{tabular}


La flora nativa de isla Kalau es interesante por su alto nivel de endemismo y por su importancia desde el punto de vista etnobotánico. El conocimiento de los diferentes usos dados a las plantas por los kawésqar constituye un patrimonio científico y cultural invaluable, debido a que en gran medida son estas las responsables de haber garantizado su supervivencia a lo largo del tiempo en estas tierras australes. Teniendo en cuenta que este trabajo representa una breve recopilación de las plantas utilizadas por el pueblo Kawésqar, se recomienda continuar investigando el uso dado a las plantas para todo el territorio ancestral.

\section{AGRADECIMIENTOS}

Agradecemos el financiamiento otorgado a esta investigación por el proyecto Innova CORFO 08CTU01-20 "Caracterización territorial del Parque Nacional Bernardo O'Higgins, su potencial económico, turístico, científico y cultural". El referido proyecto fue mandatado por CONAF Magallanes y ejecutado por el Centro Regional de Investigación CEQUA. También queremos expresar nuestro agradecimiento a Gabriela Paterito por acceder a una entrevista en Puerto Edén, a Carolina Galleguillos y Kary Haro, por su apoyo en el trabajo de terreno, a Marcela Durbhan por el análisis cartográfico y a Gabriel Quilahuilque por la elaboración de la Fig. 1.

\section{LITERATURA CITADA}

Aguilera, O. \& J. Tonko 2011. Guia etnogeográfica del Parque Nacional Bernardo O'Higgins. Ed. Fundación CEQUA, 140 pp.

Carrasco, JF, Casassa, G. \& A. Rivera 2002. Meteorological and climatological aspects of the Southern Patagonia Icefield. In: The Patagonian Icefields. A unique natural laboratory for environmental and climate change studies. Casassa, G., Sepúlveda, F.V. and RM Sinclair (eds). Kluwer/Plenum Publishers 29-41pp.

Chiarucci, A. \& I. Bonini 2005. Quantitative floristics as a tool for the assessment of plant diversity in Tuscan forests. Forest Ecology and Management, 212:160-170.

Clapperton, C., M.D. Sugden, D. Kaufman \& R.D. Mcculloch 1995. The Last Glaciation in Central Magellan Strait, Southernmost Chile. Quaternary Research, 44: 133-148.
Correa, N.M. (ed.). 1969. Flora Patagónica. Parte II. Colecciones Científicas del INTA. Tomo VIII. Buenos Aires. 219 pp.

Correa, N.M. (ed.). 1971. Flora Patagónica. Parte IV. Colecciones Científicas del INTA. Tomo VIII. Buenos Aires. 451 pp.

Correa, N.M. (ed.). 1978. Flora Patagónica. Parte III. Colecciones Científicas del INTA. Tomo VIII. Buenos Aires. 569 pp.

Correa, N.M. (ed.). 1984a. Flora Patagónica. Parte IV a. Colecciones Científicas del INTA. Tomo VIII. Buenos Aires. 559 pp.

Correa, N.M. (ed.). 1984b. Flora Patagónica. Parte IV b. Colecciones Científicas del INTA. Tomo VIII. Buenos Aires. 309 pp.

Correa, N.M. (ed.). 1988. Flora Patagónica. Parte V. Colecciones Científicas del INTA. Tomo VIII. Buenos Aires. 404 pp.

Correa, N.M. (ed.). 1998. Flora Patagónica. Parte I. Colecciones Científicas del INTA. Tomo VIII. Buenos Aires. 391 pp.

DGA, 1987. Balance Hídrico de Chile, Dirección General de Aguas, Santiago, Chile. Pp.

Dollenz, O. 1986. Relevamiento fitosociológicos en la Península Muñoz Gamero, Magallanes. Anales del Instituto de la Patagonia, Serie Ciencias Naturales, 16: 55-62.

Domínguez, E. 2007. Catálogo Preliminar de Gramíneas Introducidas en la Región de Magallanes, Año 10. $\mathrm{N}^{\circ}$ 1. URL: http://www.chlorischile.cl

Domínguez, E. 2010. Flora de interés etnobotánico usada por los pueblos originarios: aónikenk, selk'Nam, kawésqar, yagan y haush en la Patagonia Austral. Dominguezia, 26 (2): 19-29.

Forsythe, R.D. \& C. Mpodozis 1979. El Archipiélago Madre de Dios, Patagonia Occidental, Magallanes: rasgos generales de la estratigrafía y estructura del basamento pre Jurásico Superior. Revista Geológica de Chile, 7: 13-29.

Forsythe, R.D. \& C. Mpodozis 1983. Geología del Basamento pre-Jurásico Superior en el archipiélago Madre de Dios, Magallanes, Chile. Servicio Nacional de Geología y Minería, Boletín 39, 63 pp.

Hechenleitner, P., M.F. Gardner, P.I. Thomas, C. Echeverría, B. Escobar, P. Brownless \& C. Martínez 2005. Plantas amenazadas del Centro-Sur de Chile: Distribución, Conser- 
vación y Propagación. Universidad Austral de Chile-Royal Botanic Garden Edinburgh, Valdivia, Chile. 188 pp.

Hervé, F., C.M. Fanning \& R.J. Pankhurst 2003. Detrital Zircon Age Patterns and Provenance in the metamorphic complexes of Southern Chile. Journal of South American Earth Sciences, 16: 107-123.

Marticorena, C. \& M. Quezada 1985. Catálogo de la flora vascular de Chile. Gayana, Serie Botánica, 42 (1- 2): 1-157.

Marticorena, C. \& R. Rodríguez 1995. Flora de Chile Vol. 1. Ediciones de la Universidad de Concepción, Chile. 351 pp.

Matthei, O. 1995. Manual de las malezas que crecen en Chile. Alfabeta Impresores, Santiago. $554 \mathrm{pp}$.

Moore, D.M. 1983. Flora of Tierra del Fuego. Oswestry, Saint Louis, E.E.U.U. 369 pp.

Pisano, E. 1970. Vegetación del área de los fiordos Toro y Cóndor y Puerto Cutter Cove (Canal Jerónimo, Magallanes). Anales del Instituto de la Patagonia, (Chile) 1(1): 27-40.

Pisano, E. 1972. Comunidades vegetales del área de bahía Morris, isla Capitán Aracena, Tierra del Fuego (Parque Nacional Hernando de Magallanes). Anales del Instituto de la Patagonia, (Chile) 3(1-2): 103-130.

Qian, H. 1999. Floristic analysis of vascular plant genera of North America north México: characteristics of phytogeography. Journal of Biogeography, 26: 1307-1321.

Ramírez, C. \& C. San Martín 1993. La transformación antrópica de la vegetación de los ñadis del área Mapuche en el centro-sur de Chile. Boletín Museo Regional de la Araucania, 4(1):205-214.

Ramírez, C., M. Álvarez, A. Díaz \& G. Toledo 2006. Biodiversidad vegetal de la isla Ipún en la Reserva Nacional Las Guaitecas (Comuna de
Cisnes, XI Región, Chile). Revista Geográfica de Valparaíso, 37: 45-66.

Rodríguez, R., D. Alarcón \& J. Espejo 2009. Helechos nativos del Centro y Sur de Chile. Guía de Campo. Ed. Corporación Chilena de la Madera, Concepción, Chile, 212 pp.

Rodríguez, R., A. Marticorena \& E. Teneb 2008. Plantas vasculares de los ríos Baker y Pascua, Región de Aisén, Chile. Gayana Botánica, 65(1): 39-70.

Rozzi, R, R. Charlin, S. Ippi \& O. Dollenz 2004. Cabo de Hornos: un Parque Nacional libre de especies exóticas en el confín de América. Anales de Instituto de la Patagonia (Chile) 32:55-62.

Sepúlveda, F.A., F. Hervé, M. Calderón \& J.P. Lacassie, 2007. Petrological and geochemical characteristics of metamorphic and igneous units from the allochthonous Madre de Dios Terrane, Southern Chile. Gondwana Research, doi: 10.1016/j.gr.2007.06.004

Valle-Levinson, A., Blanco, J.L. \& M. Frangópulos 2006. Hydrography and frontogenesis in a glacial fjord off the Strait of Magellan. Ocean Dynamics, DOI: 10.1007/s10236-005-0048-8.

Villagrán, C. \& E. Barrera 2002. Helechos del archipiélago de Chiloé, Chile. CONAF, Puerto Montt. 23 pp.

Walter, K.S. \& H.J. Gillett 1998. 1997 IUCN Red List of Threatened Plants. Compiled by the World Conservation Monitoring Centre. IUCNThe World Conservation Union, Gland, Switzerland and Cambridge, UK. lxiv + 862 pp.

Zuloaga, F., O. Morrone \& M Belgrano 2011. Catálogo de las Plantas Vasculares del Cono Sur. Versión base de datos en sitio web del Instituto Darwinion, Argentina. URL: http:// www.darwin.edu.ar/Proyectos/FloraArgentina/FA.asp: Febrero 15, 2011 
